

\title{
Can new colored particles illuminate the Higgs?
}

\section{E. Bertuzzo, ${ }^{a}$ P.A.N. Machado ${ }^{a, b}$ and R. Zukanovich Funchal ${ }^{a, b}$}

${ }^{a}$ Institut de Physique Théorique, CEA-Saclay, 91191 Gif-sur-Yvette, France

${ }^{b}$ Instituto de Física, Universidade de São Paulo, C.P. 66.318, 05315-970 São Paulo, Brazil

E-mail: enrico.bertuzzo@cea.fr, accioly@if.usp.br, zukanov@if.usp.br

ABSTRACT: We analyze the behavior of Higgs to diphoton rate and Higgs gluon-gluon production cross section in minimal extensions of the Standard Model comprising new colored vector-like fermions that do not mix with the ordinary ones. We compare these information with constraints coming from electroweak precision measurements. We compute pair production cross sections for the lightest fermion and discuss the LHC bounds. Finally, we study the phenomenology of possible quarkonium states composed by these new colored fermions.

Keywords: Higgs Physics, Beyond Standard Model

ARXIV EPRINT: 1209.6359 


\section{Contents}

1 Introduction 1

2 Effects of new fermions in the Higgs production and diphoton decay 2

3 Brief description of two simple frameworks 4

3.1 Doublet-singlet model $(2+1) \quad 5$

3.2 Triplet-doublet model $(3+2) \quad 5$

3.3 Color interactions 5

4 Analysis of the viability of the models $\quad 6$

5 Signals at the LHC $\quad 8$

$\begin{array}{llr}6 & \text { Conclusion } & 13\end{array}$

A Explicit expressions for the two point functions that enter S,T and U 14

\section{Introduction}

The auspicious discovery of a Higgs-like particle reported by ATLAS [1] and CMS [2] experiments at the Large Hadron Collider (LHC) marks the dawn of a new chapter in the field of particle physics. This new particle seems to have many of the expected properties of the long awaited Standard Model (SM) Higgs. However, there are some alluring hints it might harbor new phenomena.

Analyses [3-5] of ATLAS and CMS data at 7 and $8 \mathrm{TeV}$ [6-10] combined with the Tevatron experiments data [11], seem to indicate discrepancies from the SM in the Higgs gluon-gluon production cross section as well as in its diphoton decay. The former seems to be $\sim 40 \%$ smaller while the latter $\sim 2.5$ larger than the SM prediction. While from the experimental point of view it is too early to conclude that new physics is imperative, from the theory side of view it is important to investigate what this might signify. In particular, since in the last decades the SM has been submitted to great experimental scrutiny passing all tests flawlessly it is not inconceivable that the first sign of new physics that will be observed at the LHC is manifested through loop effects.

In this paper we will assume this is the case and try to understand to what extent one can explain an increase of the $H \rightarrow \gamma \gamma$ partial decay width as well as a decrease of the Higgs gluon-gluon production cross section, by introducing the minimum number of extra charged fermion states that couple to the Higgs. Since the first measurements by the LHC experiments suggested the observed Higgs to diphoton rate was larger than the SM one $[12,13]$, a number of theoretical papers have attempted to explain this effect by 
introducing new fermions [14-24], scalars [14, 17, 25-32], a spin-2 resonance [33], in SUSY models [34-43] or other scenarios [44-48]. Some consequences to the vacuum stability of a wrong-sign gluon-gluon amplitude are discussed in [49].

In a previous paper [20] we have showed how it is possible to increase the Higgs diphoton rate by the introduction of new colorless fermions that do not mix with other SM fermions but mix among themselves. Our goal in this paper is to extend this idea to include colored particles. We will discuss the properties (masses, charges, couplings) that these new fermions have to satisfy in order to be at the same time in consonance with the currently observed $H \rightarrow \gamma \gamma$ width and with the gluon-gluon Higgs production cross section. We will consider the extra fermions in their smallest allowed $\mathrm{SU}(2)_{L} \times \mathrm{U}(1)_{Y}$ representations and assume that the additional states that will accompany these fermions in a complete model, are heavy enough to play no significant role in the Higgs sector. We will confront our results with the electroweak precision tests and discuss the production of these new particles at the LHC.

This work is organized as follows. In the section 2 we discuss, in a generic way, how the Higgs diphoton rate and the Higgs production by gluon-gluon fusion can be affected by new charged fermions that receive part of their mass from the electroweak symmetry breaking mechanism. In particular, we focus on the correlated role they may play in $\sigma(g g \rightarrow h)$ and $\Gamma(h \rightarrow \gamma \gamma)$. In section 3 we describe the two simple frameworks we will introduce for new colored vector-like fermions that do not mix with the SM ones. They will involve the smallest $\mathrm{SU}(2)_{L}$ representations of these fields. In the doublet-singlet (triplet-doublet) model we introduce two new fields: a $\mathrm{SU}(2)_{L}$ doublet (triplet) and a singlet (doublet). From the color point of view we will assume these new field can be either $\mathrm{SU}(3)_{c}$ triplets, sextets or octets. Given a fixed gauge group representation we will discuss in section 4 how these new particles can impact $\sigma(g g \rightarrow h), \Gamma(h \rightarrow \gamma \gamma)$ and the $S, T, U$ oblique parameters, as a function of their masses and couplings. This aims to outline what are the allowed properties of these colored fermion fields that might be needed to explain future LHC data. In section 5 we discuss the direct pair production of these particles at the LHC as well as the possible formation of quarkonia. Finally in section 6 we draw our conclusions.

\section{Effects of new fermions in the Higgs production and diphoton decay}

It may be fortuitous that the main production process of this new particle at the LHC is the gluon-gluon fusion and the most conspicuous signal observed up to now comes from its diphoton decay. Not only both happen at the loop level, probing quantum corrections coming from any new states carrying color or charge, moreover, these corrections are correlated for colored states.

We will consider here that the $125 \mathrm{GeV}$ particle discovered at the LHC is in fact the SM Higgs boson, $h$, a fundamental scalar transforming as part of a $\mathrm{SU}(2)_{L} \times \mathrm{U}(1)_{Y}$ doublet with the SM Higgs charge assignments and hypercharge $Y=1 / 2$. Assuming new colored fermions couple to the Higgs field, we can define the ratio between the production cross 
section including these new colored particles, $\sigma(g g \rightarrow h)$, and the SM one, $\left.\sigma(g g \rightarrow h)\right|_{\mathrm{SM}}$, as

$$
R_{\mathrm{GG}} \equiv \frac{\sigma(g g \rightarrow h)}{\left.\sigma(g g \rightarrow h)\right|_{\mathrm{SM}}} \approx\left|1-9.46 \sum_{i} \frac{I_{i}(R)}{Q_{i}^{2} D_{i}(R)} \delta_{c}^{i}\right|^{2},
$$

where $I_{i}(R)$ and $D_{i}(R)$ are, respectively, the index and dimension of the $\mathrm{SU}(3)_{c}$ representation of these new fermions, $Q_{i}$ their electric charge and $\delta_{c}^{i}$ parametrizes their contributions to the $h G G$ vertex and is related to the $h \rightarrow \gamma \gamma$ rate as will be discussed below. Here we have only considered the top quark in estimating the SM cross section.

Allowing for new charged uncolored fermions, as well as new colored ones, to couple to $h$, we can also define the ratio between the $h \rightarrow \gamma \gamma$ rate including this new states, $\Gamma(h \rightarrow \gamma \gamma)$, and the SM one, $\left.\Gamma(h \rightarrow \gamma \gamma)\right|_{\mathrm{SM}}$, as

$$
R_{\gamma \gamma} \equiv \frac{\Gamma(h \rightarrow \gamma \gamma)}{\left.\Gamma(h \rightarrow \gamma \gamma)\right|_{\mathrm{SM}}} \approx\left|1+\sum_{i}\left(\delta^{i}+\delta_{c}^{i}\right)\right|^{2}
$$

where $\delta^{i}$ and $\delta_{c}^{i}$ parametrize, respectively, the uncolored and colored particles new contributions with respect to the SM. To a very good approximation [50], for new fermion masses greater or of the order of the Higgs mass

$$
\begin{aligned}
\delta^{i} & =\frac{\left(Q_{i}^{\mathrm{u}}\right)^{2}}{A_{\mathrm{SM}}^{\gamma \gamma}} \frac{v}{2} \frac{d \ln M_{i}^{\mathrm{u}} 2(v)}{d v} \frac{4}{3}\left(1+\frac{7}{120} \frac{M_{h}^{2}}{M_{i}^{\mathrm{u}} 2}\right), \\
\delta_{c}^{i} & =\frac{Q_{i}^{2} D_{i}(R)}{A_{\mathrm{SM}}^{\gamma \gamma}} \frac{v}{2} \frac{d \ln M_{i}^{2}(v)}{d v} \frac{4}{3}\left(1+\frac{7}{120} \frac{M_{h}^{2}}{M_{i}^{2}}\right),
\end{aligned}
$$

where $M_{h}$ is the Higgs boson mass, $v$ is the Higgs vacuum expectation value, $A_{\mathrm{SM}}^{\gamma \gamma}$ is the main contribution to the SM Higgs to diphoton amplitude, namely, the one coming from the $\mathrm{W}$ boson and the top, i.e., $A_{\mathrm{SM}}^{\gamma \gamma}=-6.48$. Here $Q_{i}^{\mathrm{u}}\left(Q_{i}\right)$ and $M_{i}^{\mathrm{u}}\left(M_{i}\right)$ are the electric charge and masses of the new uncolored (colored) fermions. As discussed in many papers, fermion mixing allows for the new terms to have the opposite sign of the top one. This can, as we will see, increase the Higgs to diphoton rate and at the same time decrease the Higgs production through gluon-gluon fusion.

In figure 1 we show iso-contour lines of constant $R_{\gamma \gamma}$ in the plane $\delta_{c}$ versus $\delta$ (both assumed real). The SM corresponds to the point $\delta=\delta_{c}=0$ in this plot. In ref. [20] we have discussed how uncolored fermions can affect this ratio when they mix among themselves but do not mix with SM ones. This case corresponds to values of $\delta$ for which $\delta_{c}=0$, so to increase the $R_{\gamma \gamma}$ ratio only positive values of $\delta$ would be allowed. However, if one also allows for a non zero $\delta_{c}$, there are more possibilities to explain the same $R_{\gamma \gamma}$ ratio.

In figure 2 we show how the ratio $R_{G G}$ changes as a function of $\delta_{c}$ and the charge $Q$ assuming all the new particles to have common electric charge and color representation. This figure along with figure 1 can be used to understand what kind of new fermions can enter the loop diagrams in order to explain the experimental data. For instance, if we want to get a $50 \%$ decrease of the gluon-gluon production cross section with respect to the SM one, we see what are the combinations of $\delta_{c}$ and $Q$ that are allowed in all three color representations. Once we select a $\delta_{c}$, one can go back to figure 1 to see what values of $\delta$ 




Figure 1. Iso-contours of the ratio $R_{\gamma \gamma}$ in the plane $\delta_{c}$ versus $\delta$.


Figure 2. Iso-contours of the ratio $R_{G G}$ in the plane $\delta_{c}$ versus $Q$, for different $\mathrm{SU}(3)_{c}$ representations: triplets (left panel), sextets (middle panel) and octets (right panel).

are needed to explain $R_{\gamma \gamma}$. We see from these figures that to explain $R_{\gamma \gamma} \sim 2$ with only colored fermions, $\delta_{c} \sim 0.5$ is needed. This can be, in principle, achieved, in any of the three color representations, even if $R_{G G}$ deviates from the SM expectation by $\sim 10 \%$.

After this general discussion about the interplay between the effect of new colored fermions in $R_{G G}$ and $R_{\gamma \gamma}$ let us now discuss some specific realizations.

\section{Brief description of two simple frameworks}

As the addition of a sequential fourth fermion generation to the particle content of the SM is now ruled out by the LHC data at the $5 \sigma$ level [51], we will assume the new colored fermions to be vector-like. Moverover, these new particles will not mix with the SM fermions, but will couple to the Higgs and the gauge sector respecting the SM symmetry group. One 
can envisage a new quantum number in connection to an unbroken or nearly unbroken symmetry, exclusive to the new sector, in order to forbid this mixing [52].

In order to be able to build a renormalizable coupling term with the SM Higgs field and to have mixing we need to introduce at least two extra fermion fields. As in ref. [20] we will consider only the smallest allowed $\mathrm{SU}(2)_{L} \times \mathrm{U}(1)_{Y}$ representations of the new fermion states, i.e. singlets, doublets and triplets. We will also allow for these new colored fermions to be either $\mathrm{SU}(3)_{c}$ triplets, sextets or octets. The choice of the $\mathrm{SU}(3)_{c} \times \mathrm{SU}(2)_{L} \times \mathrm{U}(1)_{Y}$ representation of the new fermions will fix their couplings to the SM gauge sector. Given that, the only free parameters will be their couplings to the Higgs, their charges and their masses.

\subsection{Doublet-singlet model $(2+1)$}

The lagrangian describing the new fermion masses and couplings with the Higgs is

$$
-\mathcal{L}_{\mathrm{H}}^{2+1}=c \bar{S}_{R} H D_{L}+c \tilde{H} \bar{D}_{R} S_{L}+m_{1} \bar{D}_{R} D_{L}+m_{2} \bar{S}_{R} S_{L}+\text { h.c. },
$$

where $S$ and $D$ are, respectively, a $\mathrm{SU}(2)_{L}$ singlet and doublet field, $\tilde{H}=i \tau_{2} H^{*}, c$ is the Yukawa coupling to the Higgs, $m_{1,2}$ are the vector-like $S, D$ masses.

As usual the gauge interactions with the SM fields are described by the coupling with the SM fields and introduced via covariant derivatives [20]

$$
\mathcal{L}_{\mathrm{I}}^{2+1}=i \bar{S} \gamma^{\mu}\left(\partial_{\mu}-i g^{\prime} y B_{\mu}\right) S+i \bar{D} \gamma^{\mu}\left(\partial_{\mu}-i g W_{\mu}^{a} T^{a}-i g^{\prime} \hat{y} B_{\mu}\right) D
$$

where $g^{\prime}=e / c_{W}, g=e / s_{W}$ are the SM couplings, $s_{W}^{2}=\sin ^{2} \theta_{W}=1-c_{W}^{2} \equiv 1-M_{W}^{2} / M_{Z}^{2}$, (with $M_{Z}$ and $M_{W}$, respectively, the $\mathrm{Z}$ and $\mathrm{W}$ boson masses), and $y(\hat{y}=y-1 / 2)$ is the hypercharge of the singlet (doublet).

\subsection{Triplet-doublet model $(3+2)$}

We will consider the following mass lagrangian for the new states

$$
-\mathcal{L}_{\text {mass }}^{3+2}=c\left(\bar{D}_{R} T_{L} H+\bar{D}_{L} T_{R} H\right)+m_{1} \bar{T}_{L} T_{R}+m_{2} \bar{D}_{L} D_{R}+\text { h.c. },
$$

where $D$ and $T$ are, respectively, a $\mathrm{SU}(2)_{L}$ doublet and triplet fields, $c$ is their coupling to the SM Higgs field and $m_{1}$ and $m_{2}$ their vector-like masses.

The gauge interactions with the SM fields are described again in the usual way by [20]

$$
\mathcal{L}_{\mathrm{I}}^{3+2}=i \bar{D} \gamma^{\mu}\left(\partial_{\mu}-i g W_{\mu}^{a} T^{a}-i g^{\prime} y B_{\mu}\right) D+i \bar{T} \gamma^{\mu}\left(\partial_{\mu}-i g W_{\mu}^{a} T^{a}-i g^{\prime} \hat{y} B_{\mu}\right) T,
$$

here $y(\hat{y}=y-1 / 2)$ is the hypercharge of the doublet (triplet).

\subsection{Color interactions}

Independent of their $\mathrm{SU}(2)_{L}$ representations our colored fermions will couple to gluons according to their $\mathrm{SU}(3)_{c}$ representation. The strong sector lagrangian can be written as

$$
\mathcal{L}_{\mathrm{S}}=\sum_{i} \bar{\psi}_{i} i \gamma^{\mu}\left(\partial_{\mu}-i g_{s} t^{A} G_{\mu}^{A}\right) \psi_{i}
$$


where $g_{s}$ is the strong coupling constant and the sum runs over all the mass eigenstates of the new fermions. These will be three states in the singlet-doublet model and five states in the triplet-doublet model. The dependence on the color representation is encoded in the $t^{A}$ matrices.

\section{Analysis of the viability of the models}

For each model we have investigated the possibility of the new fermions to be able to explain the current allowed regions of $R_{G G}$ and $R_{\gamma \gamma}$, according to ref. [3], and at the same type fulfill the requirements of the electroweak precision tests.

New electroweak states necessarily contribute to the vacuum polarization amplitudes of the electroweak gauge bosons $\Pi_{a b}^{\mu \nu}\left(q^{2}\right)=-i g^{\mu \nu} \Pi_{a b}\left(q^{2}\right)+q^{\mu} q^{\nu}$ terms [53, 54]. The new effects can be parametrized by the so-called quantum oblique parameters $S, T$ and $U$ defined as [54]

$$
\begin{aligned}
& \alpha\left(M_{Z}^{2}\right) S^{\mathrm{NP}}=\frac{4 s_{W}^{2} c_{W}^{2}}{M_{Z}^{2}}\left[\Pi_{Z Z}^{\mathrm{NP}}\left(M_{Z}^{2}\right)-\Pi_{Z Z}^{\mathrm{NP}}(0)-\Pi_{\gamma \gamma}^{\mathrm{NP}}\left(M_{Z}^{2}\right)-\frac{c_{W}^{2}-s_{W}^{2}}{c_{W} s_{W}} \Pi_{\gamma Z}^{\mathrm{NP}}\left(M_{Z}^{2}\right)\right], \\
& \alpha\left(M_{Z}^{2}\right) T^{\mathrm{NP}}= \frac{\Pi_{W W}^{\mathrm{NP}}(0)}{M_{W}^{2}}-\frac{\Pi_{Z Z}^{\mathrm{NP}}(0)}{M_{Z}^{2}}, \\
& \alpha\left(M_{Z}^{2}\right) U^{\mathrm{NP}}=4 s_{W}^{2}\left[\frac{\Pi_{W W}^{\mathrm{NP}}\left(M_{W}^{2}\right)-\Pi_{W W}^{\mathrm{NP}}(0)}{M_{W}^{2}}-c_{W}^{2}\left(\frac{\Pi_{Z Z}^{\mathrm{NP}}\left(M_{Z}^{2}\right)-\Pi_{Z Z}^{\mathrm{NP}}(0)}{M_{Z}^{2}}\right)\right. \\
&\left.-2 s_{W} c_{W} \frac{\Pi_{\gamma Z}^{\mathrm{NP}}\left(M_{Z}^{2}\right)}{M_{Z}^{2}}-s_{W}^{2} \frac{\Pi_{\gamma \gamma}^{\mathrm{NP}}\left(M_{Z}^{2}\right)}{M_{Z}^{2}}\right],
\end{aligned}
$$

the explicit expressions in terms of our parameters can be found in the appendix.

The most recent electroweak study of the SM observables, finds the following best fit values for the oblique parameters [55]

$$
\begin{aligned}
& \Delta S=S-S_{\mathrm{SM}}=0.03 \pm 0.10 \\
& \Delta T=T-T_{\mathrm{SM}}=0.05 \pm 0.12 \\
& \Delta U=U-U_{\mathrm{SM}}=0.03 \pm 0.10
\end{aligned}
$$

for the reference Higgs and top masses $M_{H \text {,ref }}=126 \mathrm{GeV}$ and $m_{t, \text { ref }}=173 \mathrm{GeV}$, with the associated correlation matrix

$$
V=\left(\begin{array}{ccc}
1 & +0.89 & -0.54 \\
+0.89 & 1 & -0.83 \\
-0.54 & -0.83 & 1
\end{array}\right)
$$

As in ref. [20], we will include these constraints in our models by minimizing the $\chi^{2}$ function

$$
\chi^{2}=\sum_{i, j}\left(X_{i}^{\mathrm{NP}}-X_{i}\right)\left(\sigma^{2}\right)_{i j}^{-1}\left(X_{j}^{\mathrm{NP}}-X_{j}\right)
$$

where $X_{i}=\Delta S, \Delta T, \Delta U$, are the fitted values of the oblique parameters with their corresponding uncertainties $\sigma_{i}$ defined in eq. (4.2), $X_{i}^{\mathrm{NP}}=S^{\mathrm{NP}}, T^{\mathrm{NP}}, U^{\mathrm{NP}}$ are the contributions 

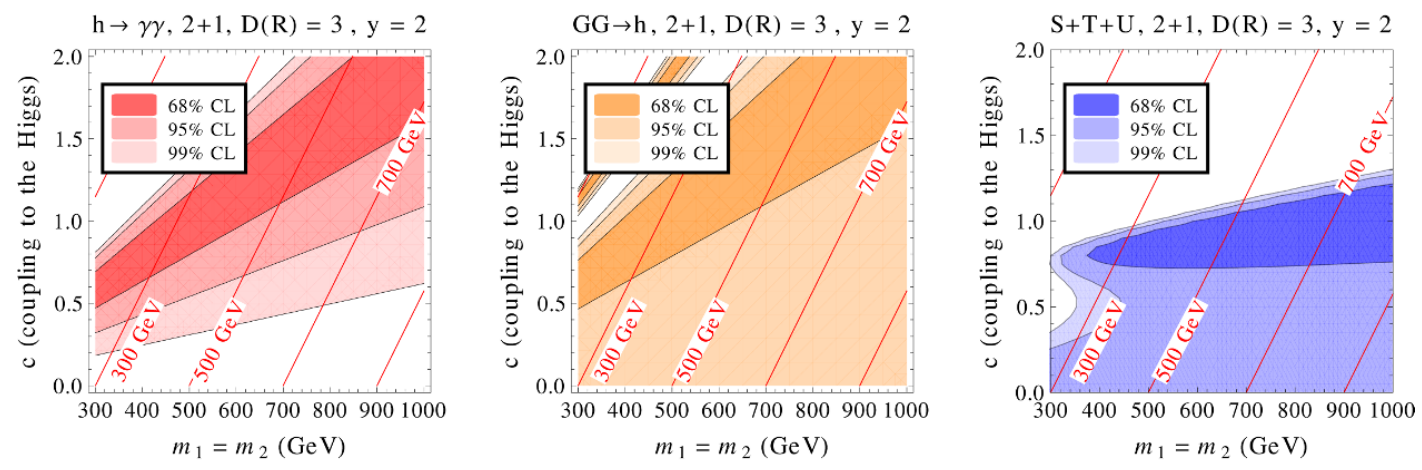

Figure 3. Allowed region in the plane $m_{1}=m_{2}$ and $c$ for the $2+1$ model with hypercharge $y=2$ and color triplet fermions $(\mathrm{D}(\mathrm{R})=3)$ for $R_{\gamma \gamma}$ (left panel), $R_{G G}$ (middle panel) and the oblique parameters $S, T$ and $U$ (right panel). The allowed regions for $R_{\gamma \gamma}$ and $R_{G G}$ are taken from ref. [3]. We also show the iso-lines of constant mass for the lightest colored fermion (in red).

from the extra states and $\left(\sigma_{i j}\right)^{2} \equiv \sigma_{i} V_{i j} \sigma_{j}$. We will allow the values of the parameters of our models to vary such that $\Delta \chi^{2}=(3.53,7.81,11.3)$, which correspond to $(68 \%, 95 \%, 99 \%)$ CL for a three-parameter fit. In a complete model there will be other states that appear in conjunction with the new colored fermions we are considering in this work. In principle, these particles also contribute to the vacuum polarization amplitudes. Having said that, we will here assume these other particles are sufficiently heavy to play no consequent role.

To illustrate the behavior of $R_{\gamma \gamma}, R_{G G}$ and $S, T$ and $U$ combined in the models with colored fermions as a function of the parameters $m_{1}=m_{2}$ and $c$, in figure 3 we show, in the case of triplet colored fermions in the $2+1$ model with $y=2$, the allowed regions for $R_{\gamma \gamma}$ (left panel), $R_{G G}$ (middle panel) and $S, T$ and $U$ combined (right panel). We can observe that in this example it is possible to explain $R_{\gamma \gamma}$ and $R_{G G}$, even in the current $68 \%$ CL allowed region and still be compatible with the oblique parameters. In figure 4 we show the same three panels for the triplet colored fermions in the $3+2$ model with $y=-5 / 2$. Here the tension with the oblique parameters is apparent and mainly driven by $S$. In this case it is quite difficult to explain the experimental ratios and at the same time satisfy the electroweak tests, unless $R_{\gamma \gamma}$ and $R_{G G}$ turn out to be respectively lower and higher than the present best fit value, i.e. closer to their SM predictions.

Looking at figures 5 and 6 we can have a better idea of values of the model parameters $m_{1}=m_{2}$ and $c$ which may be needed to satisfy experimental requirements in the near future, as the situation of Higgs production and diphoton decay get settled by the accumulation of LHC data.

In figure 5 we show the allowed region at $68 \%, 95 \%$ and $99 \%$ CL for the $2+1$ model parameters that satisfy $S, T$ and $U$ combined. Panels on the left are for $\mathrm{SU}(3)_{c}$ triplets, on the middle for $\mathrm{SU}(3)_{c}$ sextets and on the right for $\mathrm{SU}(3)_{c}$ octets. Panels from top to bottom are, respectively, for $y=1,2$ and 3 . We also show in these plots iso-lines of constant $R_{\gamma \gamma}$ (dashed red) and constant $R_{G G}$ (solid orange). We see that for all the $\mathrm{SU}(3)_{c}$ representations it is difficult to explain $R_{\gamma \gamma} \sim 1.5-2$ with $y=1$ because of the new 

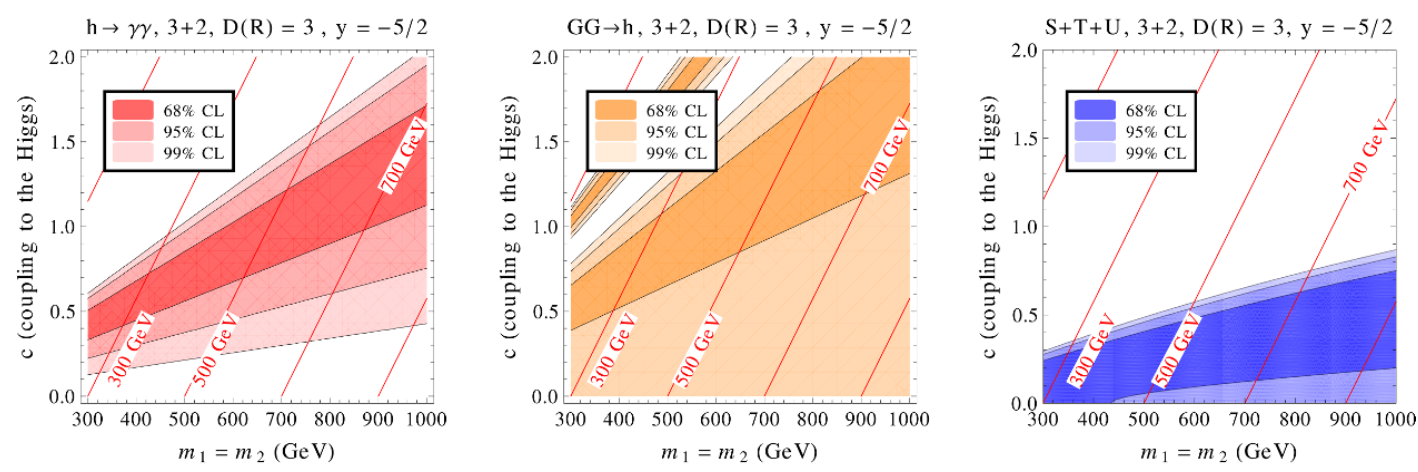

Figure 4. Allowed region in the plane $m_{1}=m_{2}$ and $c$ for the $3+2$ model with hypercharge $y=-5 / 2$ and color triplet fermions $(\mathrm{D}(\mathrm{R})=3)$ for $R_{\gamma \gamma}$ (left panel), $R_{G G}$ (middle panel) and the oblique parameters $S, T$ and $U$ (right panel). The allowed regions for $R_{\gamma \gamma}$ and $R_{G G}$ are taken from ref. [3]. We also show the iso-lines of constant mass for the lightest colored fermion (in red).

fermions contributions to the oblique parameters. However an increase of $10-20 \%$ is quite feasible. At the same time $R_{G G} \gtrsim 0.5-0.75$ is also possible, in particular, for colored sextets and octets. For $y=2$ it is possible to explain $R_{\gamma \gamma}$ as high as $\sim 1.5-2$ and at the same time $R_{G G} \gtrsim 0.55$. This is true for color triplets, sextets and octets. For $y=3$, it is easy to explain $R_{\gamma \gamma}>1$, nevertheless for color representations higher than triplet the increase in the ratio allowed by the oblique parameters might be too much, unless one goes to higher masses. Also, except for the triplet case, it is difficult to accommodate $R_{G G}$ lower than approximately 0.6 or so. For colored sextets and octets, it is possible to explain $R_{G G} \sim 0.5$, or even lower, but this requires lower masses.

In figure 6 we now show the $68 \%, 95 \%$ and $99 \%$ CL allowed for the $3+2$ model parameters that satisfy $S, T$ and $U$ combined. Again, panels on the left are for $\mathrm{SU}(3)_{c}$ triplets, on the middle for $\mathrm{SU}(3)_{c}$ sextets and on the right for $\mathrm{SU}(3)_{c}$ octets. Panels on the first, second, third and fourth rows are, respectively, for $y=-5 / 2,-1 / 2,1 / 2$ and $5 / 2$. We observe that independently of the $\mathrm{SU}(3)_{c}$ representation, it is possible to get $R_{\gamma \gamma} \sim 1.1-1.5$ $\left(R_{\gamma \gamma} \sim 1.1-1.25\right)$ if $y=-5 / 2(y=1 / 2)$. However, to get $R_{G G} \sim 0.5-0.75$ one need to have $|y| \lesssim 1 / 2$. For color triplets we have checked this can also be achieved for $|y| \lesssim 3 / 2$.

One final remark is in order. When $c \rightarrow 0$ for $m_{1}=m_{2}$, all the masses are degenerate, so that for both models $\Delta S, \Delta T \rightarrow 0$. Hence, the allowed regions at $1 \sigma$ that appear in some panels, as for instance in the central bottom panel of figure 5 or in all panels in the two last rows of figure 6 , are driven by $\Delta U$.

\section{Signals at the LHC}

We now come to the discussion of the direct production at the LHC. We have calculated the pair production cross section of the lightest colored fermion of each model. This was done for p-p collisions at the LHC running at a center of mass energy of $8 \mathrm{TeV}$, using MadGraph5 [56] with CTEQ6L [57] parton distribution functions for the proton and imposing the following loose cuts: $-2.5<\eta<2.5$ and $p_{T}>20 \mathrm{GeV}$ for each fermion. 

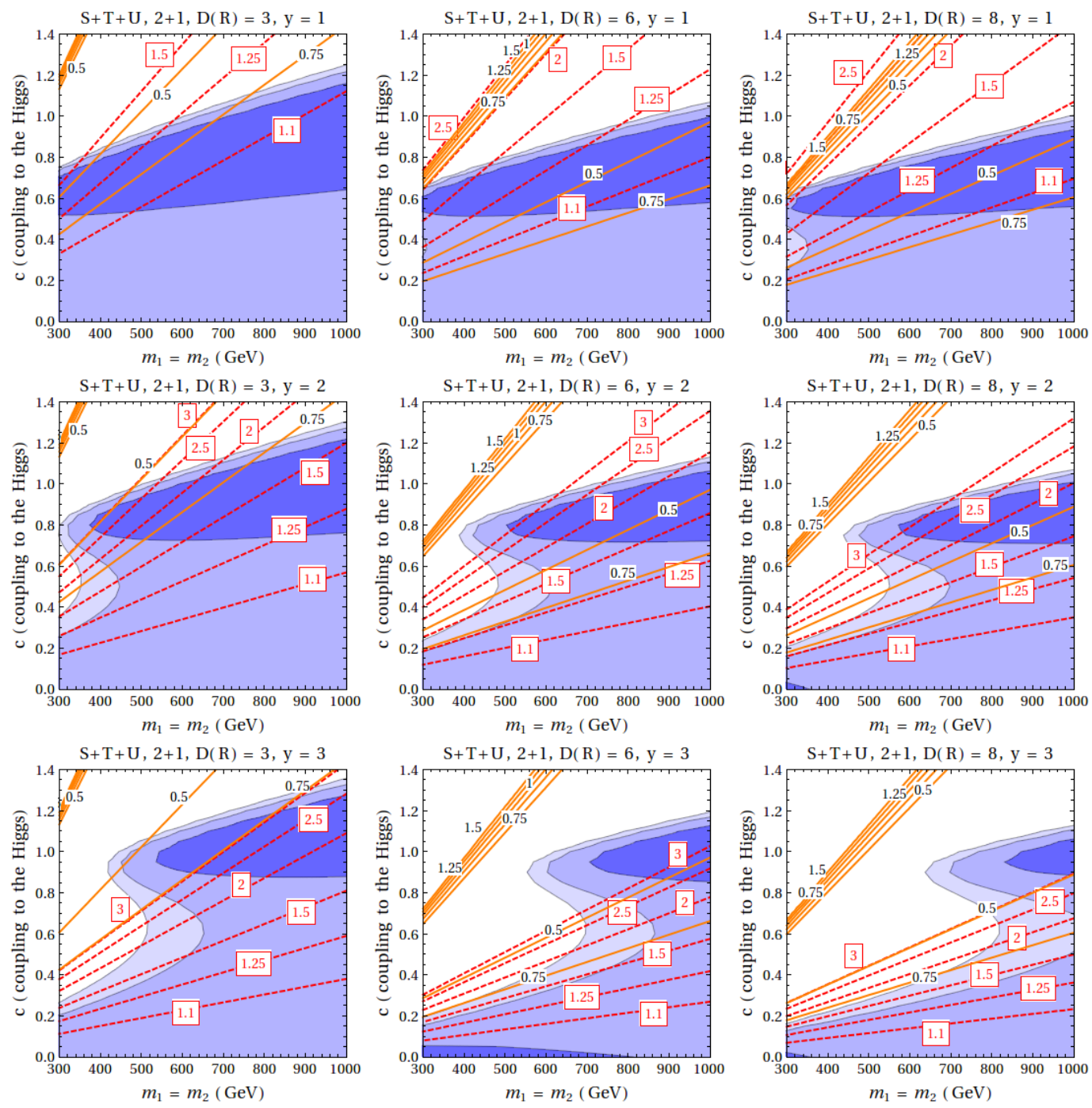

Figure 5. Allowed regions in the plane $m_{1}=m_{2}$ and $c$ for combined $S, T$ and $U$ at $68 \%, 95 \%$ and $99 \%$ CL in the $2+1$ model. Panels on the left are for $\mathrm{SU}(3)_{c}$ triplets, on the middle for $\mathrm{SU}(3)_{c}$ sextets and on the right for $\mathrm{SU}(3)_{c}$ octets. Panels from top to bottom are, respectively, for $y=1,2$ and 3. We also show iso-lines of constant $R_{\gamma \gamma}$ (dashed red) and $R_{G G}$ (solid orange).

For all models, the main contributions to pair production of the lightest particle come from gluon-gluon diagrams so that just a marginal dependence on the hypercharge (if any) is expected.

In figure 7 we show the pair production cross section for the lightest particle of the triplet $2+1$ model with $y=2$ as a function of $m_{1}=m_{2}$ and the coupling to the Higgs (c) for various masses of the lightest particle. We have confirmed that this cross section changes at the most by a few per cent for other values of $y$ in the $2+1$ model. This is also true for the triplet $3+2$ model, so we do not explicit show a plot for this case. This cross section, nevertheless depends on the color representation. When going from the triplet to the sextet or octet, the gluon-gluon contribution increases by a factor of 20 , 



Figure 6. Allowed regions in the plane $m_{1}=m_{2}$ and $c$ for combined $S, T$ and $U$ at $68 \%, 95 \%$ and $99 \%$ CL in the $3+2$ model. Panels on the left are for $\mathrm{SU}(3)_{c}$ triplets, on the middle for $\mathrm{SU}(3)_{c}$ sextets and on the right for $\mathrm{SU}(3)_{c}$ octets. Panels on the first, second, third and fourth rows are, respectively, for $y=-5 / 2,-1 / 2,1 / 2$ and $5 / 2$. We also show iso-lines of constant $R_{\gamma \gamma}$ (dashed red) and $R_{G G}$ (solid orange). 


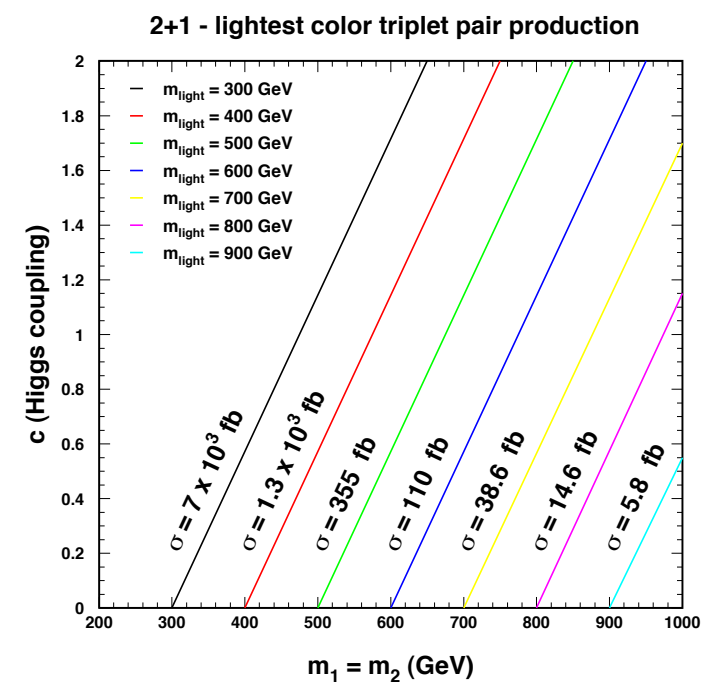

Figure 7. Pair production cross section for various masses of the lightest $\mathrm{SU}(3)_{c}$ triplet for the $2+1$ model as a function of the coupling to the Higgs $(c)$ and the vector-like mass $m_{1}=m_{2}$. Here $y=2$.

while the $q \bar{q}$ contribution increases by a factor of $5-6$. Due to the difference in parton distribution function among gluons and quarks, the relative importance between the gluongluon and the $q \bar{q}$ contributions to the total cross section will depend on the mass of the lightest fermion.

These new fermions can also form superheavy-quarkonia. Although the calculation performed for the production and decays of quarkonium in ref. [58] assumed a fourth generation of heavy chiral quarks, we can use their results to estimate the production and decay of the lightest of the heavy bound states. Production and decay of quarkonium states depend crucially on the quarkonium wave function which requires a choice of the interquark potential model. There are several possible choices for this potential model. For our purposes, and for simplicity, we will assume a Coulomb potential

$$
V(r)=-\frac{4}{3} \frac{\alpha_{s}\left(m_{Q}^{2}\right)}{r},
$$

with the running strong coupling $\alpha_{s}$ calculated at the scale $m_{Q}$, the mass of the new colored state. Notice that the factor of $4 / 3$ applies only to color triplet, while in general $\frac{4}{3} \rightarrow C_{R}-\frac{1}{2} C_{\mathcal{R}}$ [59], with $C_{R}, \mathcal{R}$ the quadratic Casimirs of the constituent fermion and of the bound state respectively. In what follows, we will use the triplet case as representative.

The quarkonium radial wave function squared for the $\mathrm{S}$ state and the derivative for the $\mathrm{P}$ state at the origin $(r=0)$ can be derived from the potential as

$$
\begin{aligned}
\left|R_{S}(0)\right|^{2} & =m_{Q}\left\langle\frac{d V}{d r}\right\rangle \\
\left|R_{P}(0)^{\prime}\right|^{2} & =\frac{m_{Q}}{9}\left\langle\frac{1}{r^{2}} \frac{d V}{d r}+4 \frac{(E-V)}{r^{3}}\right\rangle,
\end{aligned}
$$

where $\langle.$.$\rangle denotes the expectation value, E=M_{X}-2 m_{Q}$ (with $M_{X}$ and $m_{Q}$ the mass of the quarkonium and of the constituent heavy fermion, respectively), so that $\left|R_{S}(0)\right|^{2}=$ 
$4\left(2 \alpha_{s} m_{Q} / 3\right)^{3}$ and $\left|R_{P}(0)^{\prime}\right|^{2}=\left(2 \alpha_{s} m_{Q} / 3\right)^{5} / 24$. According to [58] this approximation underestimates $\left|R_{S}(0)\right|^{2}$ by less than a factor 2 , and $\left|R_{P}^{\prime}(0)\right|^{2}$ by a factor 10 or so, but this should be enough for our purposes.

We will only consider here the quarkonium bound states with smallest spin: the pseudoscalar $\eta\left(0^{-+}\right)$and the scalar $\chi\left(0^{++}\right)$. They can be directly produced via the gluon-gluon fusion mechanism. Their partial widths into gluons, which dominate by far the decays of the $\eta(\chi)$ are

$$
\begin{aligned}
\Gamma(\eta \rightarrow g g) & =\frac{8 \alpha_{s}^{2}\left(m_{Q}^{2}\right)}{3 M_{\eta}^{2}}\left|R_{S}(0)\right|^{2}, \\
\Gamma(\chi \rightarrow g g) & =\frac{96 \alpha_{s}^{2}\left(m_{Q}^{2}\right)}{M_{\chi}^{4}}\left|R_{P}(0)^{\prime}\right|^{2},
\end{aligned}
$$

so that $\Gamma(\eta \rightarrow g g) \sim \mathcal{O}(\mathrm{MeV})$ and $\Gamma(\chi \rightarrow g g) \sim \mathcal{O}(\mathrm{keV})$, for $M_{\eta}=M_{\chi} \sim 1 \mathrm{TeV}$. In both cases we take $m_{Q}=500 \mathrm{GeV}$.

The production cross section for a spin- 0 quarkonium $X$ via gluon-gluon fusion at the LHC can be written in terms of its gluonic decay width as

$$
\sigma(p p \rightarrow g g \rightarrow X)=\frac{\pi^{2} \tau}{8 M_{X}^{3}} \Gamma(X \rightarrow g g) \int_{\tau}^{1} \frac{d x}{x} g\left(x, q^{2}\right) g\left(\tau / x, q^{2}\right),
$$

where $\tau=M_{X}^{2} / s, s=4 E_{\mathrm{cm}}^{2}, g\left(x, q^{2}\right)$ is the gluon parton distribution function for the proton. For $M_{\eta}=M_{\chi} \sim 1 \mathrm{TeV}$, we have estimated $\sigma(p p \rightarrow \eta) \sim 0.2 \mathrm{fb}$ and $\sigma(p p \rightarrow \chi) \sim$ $2 \times 10^{-4} \mathrm{fb}$ for the LHC at a center of mass energy of $8 \mathrm{TeV}$. Assuming $\operatorname{BR}(\eta, \chi \rightarrow g g) \simeq 1$, these results can be compared to current experimental limits on dijets signal cross section at the LHC, which for $M_{\eta, \chi} \sim 1 \mathrm{TeV}$ and $7 \mathrm{TeV}$ center of mass energy, read $\sigma_{j j} \lesssim 4 \mathrm{pb}$ [59]. Note that the Tevatron also has a limit of $\sigma_{j j} \lesssim 200 \mathrm{fb}$ [60] for a $1 \mathrm{TeV}$ mass.

In general, the available experimental limits on new charged colored particles cannot be directly applied in a model independent way. In fact, a complete model featuring the new colored fermions may require or lead to a very small mixing with the standard model fermions. If such a mixing is small enough such that the decay is prompt, then one could expect that the searches in the LHC for a heavy top-like or bottom-like quarks can place limits on the lightest color triplet. At the moment, the searches in the LHC for a heavy top-like quark, $t^{\prime}$, that decays as $t^{\prime} \rightarrow b W$, or $t^{\prime} \rightarrow t Z$ place the limit $M_{t^{\prime}} \gtrsim$ $415-557 \mathrm{GeV}$ at $95 \% \mathrm{CL}[61,62]$. Also searches by CMS for $b^{\prime}$-quarks that are assumed to decay exclusively as $b^{\prime} \rightarrow t W$ excludes the existence of these quarks for $M_{b^{\prime}}$ below $611 \mathrm{GeV}$ [63]. On the other hand, if those particles are long-lived, having a lifetime higher than $10 \mu \mathrm{s}$, the limits on long-lived particles at the LHC should apply. Searches for longlived supersymmetric particles constrain gluinos and stops to have masses roughly above $900 \mathrm{GeV}$ and $600 \mathrm{GeV}$ [64], respectively. In case the lifetime of those particles is also smaller than $1000 \mathrm{~s}$, searches for stopped long-lived particles also play a role, ruling out gluinos and stops with masses below $640 \mathrm{GeV}$ and $340 \mathrm{GeV}$ at $95 \% \mathrm{CL}$, respectively [65]. In our case, we expect the color triplet and octet to have production cross sections somewhat similar to stops and gluinos, and hence although the bounds are not directly applicable, 
the order of magnitude should be the same. In summary, we expect that masses below $\mathcal{O}(400-900) \mathrm{GeV}$ are excluded depending on the details, such as the stopping probability, the efficiency of charge measurement or the hadronization model used in the analysis. This certainly pushes the allowed parameter space to higher vectorial masses. Finally, one could envisage the second lightest particle decaying weakly into the lightest one, increasing the production of $W$ bosons at the LHC. For this to impose a bound, the mass of the second lightest fermion should be light enough to be significantly produced at the LHC. See e.g. ref. [66].

\section{Conclusion}

We have investigated how new colored vector-like fermions, in the smallest representations of $\mathrm{SU}(3)_{c} \times \mathrm{SU}(2)_{L} \times \mathrm{U}(1)_{Y}$, can contribute to the Higgs to diphoton rate and also modify the main Higgs production cross section. We assume these new particles do not mix with the SM fermions, but since they acquire part of their mass from the electroweak symmetry breaking mechanism, they mix among themselves.

For concreteness we have analyzed two minimal models with respect to the $\mathrm{SU}(2)_{L}$ representation of the fermion fields: the doublet-singlet and the triplet-doublet model. In both cases we have supposed the colored fermions to be either in the triplet, sextet or octect representation of $\mathrm{SU}(3)_{c}$. We have established what are the values of the parameters of the models (coupling to the Higgs, charges and vector-like masses) that can increase/decrease $\Gamma(h \rightarrow \gamma \gamma)$ and $\sigma(g g \rightarrow h)$ and still be consistent with the electroweak tests by fitting the model to the current bounds on $S, T$ and $U$.

Many of the combined analysis of the available Higgs boson experimental results $[3,4]$ seem to proclaim $h \rightarrow \gamma \gamma$ and $g g \rightarrow h$ data already point to the need of physics beyond the SM. The ATLAS Collaboration [67] also points towards $\Gamma(h \rightarrow \gamma \gamma) \sim 1.4 \Gamma(h \rightarrow \gamma \gamma)_{\mathrm{SM}}$ and $\sigma(g g \rightarrow h) \sim 1.3 \sigma(g g \rightarrow h)_{\mathrm{SM}}$, but both consistent with the SM at $95 \%$ CL.

Ultimately, only more LHC data will tell us if the Higgs to diphoton rate and/or the Higgs production cross section through gluon-gluon fusion are in fact a door to new physics, or if they can be perfectly understood in the SM context. If either one turns out to be away from SM expectations we provide in this paper some possible scenarios that can be experimentally testable.

\section{Acknowledgments}

This work was supported by Fundação de Amparo à Pesquisa do Estado de São Paulo (FAPESP), Conselho Nacional de Desenvolvimento Científico e Tecnológico (CNPq), by the European Commission under the contract PITN-GA-2009-237920 and by the Agence National de la Recherche under contract ANR 2010 BLANC 0413 01. We thank O.J.P. Éboli for useful comments and discussions, and L. G. Almeida for discussions in the early stages of the project. We also thank Y. Kats for useful comments and for pointing out ref. [59]. 


\section{A Explicit expressions for the two point functions that enter S,T and U}

We can write the two point functions that enter in the oblique parameters defined in eq. (4.1) as

$$
\Pi_{V_{1} V_{2}}^{\mathrm{NP}}(s)=2 g_{V_{1}}^{\alpha \beta} g_{V_{2}}^{\alpha \beta}\left[\Pi_{V+A}\left(s, M_{\alpha}, M_{\beta}\right)+\Pi_{V-A}\left(s, M_{\alpha}, M_{\beta}\right)\right],
$$

where a sum is implicit over all fermions involved in the loop, $V_{1}, V_{2}=W, Z, \gamma$, the physical masses of the fermions $f_{\alpha}$ and $f_{\beta}$ are $M_{\alpha}$ and $M_{\beta}$, respectively, their couplings with the gauge bosons are $g_{V_{1}, V_{2}}^{\alpha \beta}$ and

$$
\begin{aligned}
\Pi_{V+A}\left(s, M_{\alpha}, M_{\beta}\right)= & \frac{N_{c}}{24 \pi^{2}}\left[M_{\alpha}^{2} \ln M_{\alpha}^{2}\left(1-\frac{\left(M_{\alpha}^{2}-M_{\beta}^{2}\right)}{2 s}\right)+M_{\beta}^{2} \ln M_{\beta}^{2}\left(1-\frac{\left(M_{\beta}^{2}-M_{\alpha}^{2}\right)}{2 s}\right)\right. \\
& -\frac{s}{3}+\frac{\left(M_{\alpha}^{2}-M_{\beta}^{2}\right)^{2}}{2 s}+\left(s-\frac{\left(M_{\alpha}^{2}+M_{\beta}^{2}\right)}{2}-\frac{\left(M_{\alpha}^{2}-M_{\beta}^{2}\right)^{2}}{2 s}\right) \\
& \left.\times\left(\bar{B}_{0}\left(s, M_{\alpha}, M_{\beta}\right)-\ln \left(M_{\alpha} M_{\beta}\right)\right)+\Delta_{\text {div }}\right]
\end{aligned}
$$

and

$$
\Pi_{V-A}\left(s, M_{\alpha}, M_{\beta}\right)=\frac{N_{c}}{8 \pi^{2}} M_{\alpha} M_{\beta}\left(\bar{B}_{0}\left(s, M_{\alpha}, M_{\beta}\right)-\ln \left(M_{\alpha} M_{\beta}\right)+\Delta_{\epsilon}\right),
$$

here $N_{c}$ are the number of colors, the divergent part $\Delta_{\text {div }} \equiv \Delta_{\epsilon}\left(s-\frac{3}{2}\left(M_{\alpha}^{2}+M_{\beta}^{2}\right)\right)$ with $\Delta_{\epsilon}=\frac{2}{\epsilon}-\gamma+\ln 4 \pi+\ln \mu^{2}$.

We have used the finite part of the $B_{0}$ function

$$
\bar{B}_{0}\left(s, M_{\alpha}, M_{\beta}\right)=1-\frac{M_{\alpha}^{2}+M_{\beta}^{2}}{M_{\alpha}^{2}-M_{\beta}^{2}} \ln \left(\frac{M_{\alpha}}{M_{\beta}}\right)+F\left(s, M_{\alpha}, M_{\beta}\right),
$$

with

$F\left(s, M_{\alpha}, M_{\beta}\right)=-1+\frac{M_{\alpha}^{2}+M_{\beta}^{2}}{M_{\alpha}^{2}-M_{\beta}^{2}} \ln \left(\frac{M_{\alpha}}{M_{\beta}}\right)-\int_{0}^{1} d x \ln \left(\frac{x^{2}-x\left(s+M_{\alpha}^{2}-M_{\beta}^{2}\right)+M_{\alpha}^{2}-i \epsilon}{M_{\alpha} M_{\beta}}\right)$.

In the doublet-singlet model the physical masses are

$$
M_{1,2}=\frac{1}{2}\left[\left(m_{1}+m_{2}\right) \mp \sqrt{\left(m_{2}-m_{1}\right)^{2}+4 c^{2} v^{2}}\right], \quad M_{3}=m_{1},
$$

and the only non zero couplings are

$$
\begin{array}{ll}
g_{\gamma}^{11}=g_{\gamma}^{22}=e\left(\hat{y}+\frac{1}{2}\right), & g_{\gamma}^{33}=e\left(\hat{y}-\frac{1}{2}\right), \\
g_{W}^{13}=\frac{g}{\sqrt{2}} \sin \theta, & g_{W}^{23}=\frac{g}{\sqrt{2}} \cos \theta,
\end{array}
$$




$$
\begin{aligned}
& g_{Z}^{11}=-g^{\prime} s_{W}\left(\hat{y}+\frac{1}{2}\right) \cos ^{2} \theta+\left(\frac{g}{2} c_{W}-g^{\prime} s_{W} \hat{y}\right) \sin ^{2} \theta, \\
& g_{Z}^{22}=-g^{\prime} s_{W}\left(\hat{y}+\frac{1}{2}\right) \sin ^{2} \theta+\left(\frac{g}{2} c_{W}-g^{\prime} s_{W} \hat{y}\right) \cos ^{2} \theta, \\
& g_{Z}^{33}=-\hat{y} g^{\prime} s_{W}-\frac{g}{2} c_{W}, \\
& g_{Z}^{12}=g_{Z}^{21}=\left(g^{\prime} s_{W}\left(\hat{y}+\frac{1}{2}\right)+\frac{g}{2} c_{W}-g^{\prime} s_{W} \hat{y}\right) \cos \theta \sin \theta,
\end{aligned}
$$

with

$$
\cos \theta=\frac{\left(m_{2}-m_{1}-B\right)}{\sqrt{4 c^{2} v^{2}+\left(m_{2}-m_{1}-B\right)^{2}}}, \quad B=\sqrt{\left(m_{2}-m_{1}\right)^{2}+4 c^{2} v^{2}} .
$$

In the triplet-doublet model the physical masses are

$$
\begin{aligned}
M_{1,2} & =\frac{1}{2}\left[\left(m_{1}+m_{2}\right) \mp \sqrt{\left(m_{2}-m_{1}\right)^{2}+4 c^{2} v^{2}}\right], \\
M_{3,4} & =\frac{1}{2}\left[\left(m_{1}+m_{2}\right) \mp \sqrt{\left(m_{2}-m_{1}\right)^{2}+2 c^{2} v^{2}}\right], \\
M_{5} & =m_{1},
\end{aligned}
$$

and the only non zero couplings are

$$
\begin{array}{rlrl}
g_{\gamma}^{11}=g_{\gamma}^{22}=e(\hat{y}+1), & g_{\gamma}^{33}=e(\hat{y}-1), \quad g_{\gamma}^{44}=g_{\gamma}^{55}=e \hat{y} \\
g_{W}^{14}=g\left(\frac{1}{\sqrt{2}} \cos \theta \cos \theta^{\prime}+\sin \theta \sin \theta^{\prime}\right), & g_{W}^{15}=g\left(\sin \theta \cos \theta^{\prime}-\frac{1}{\sqrt{2}} \cos \theta \sin \theta^{\prime}\right), \\
g_{W}^{24}=g\left(\cos \theta \sin \theta^{\prime}-\frac{1}{\sqrt{2}} \sin \theta \cos \theta^{\prime}\right), & g_{W}^{25}=g\left(\cos \theta \cos \theta^{\prime}+\frac{1}{\sqrt{2}} \sin \theta \sin \theta^{\prime}\right), \\
g_{W}^{43}=g \sin \theta^{\prime}, & g_{W}^{53}=g \cos \theta^{\prime}, \\
g_{Z}^{11}=\left(\frac{g}{2} c_{W}-y g^{\prime} s_{W}\right) \cos ^{2} \theta+\left(g c_{W}-\hat{y} g^{\prime} s_{W}\right) \sin ^{2} \theta, \\
g_{Z}^{22}=\left(\frac{g}{2} c_{W}-y g^{\prime} s_{W}\right) \sin ^{2} \theta+\left(g c_{W}-\hat{y} g^{\prime} s_{W}\right) \cos ^{2} \theta \\
g_{Z}^{33}=-\hat{y} g^{\prime} s_{W}-g c_{W}, \\
g_{Z}^{44}=\left(-\frac{g}{2} c_{W}-y g^{\prime} s_{W}\right) \cos ^{2} \theta^{\prime}-\hat{y} g^{\prime} s_{W} \sin ^{2} \theta^{\prime}, \\
g_{Z}^{55}=\left(-\frac{g}{2} c_{W}-y g^{\prime} s_{W}\right) \sin ^{2} \theta^{\prime}-\hat{y} g^{\prime} s_{W} \cos ^{2} \theta^{\prime}, \\
g_{Z}^{12}=g_{Z}^{21}=\left(-\frac{g}{2} c_{W}+y g^{\prime} s_{W}+g c_{W}-\hat{y} g^{\prime} s_{W}\right) \sin \theta \cos \theta, \\
g_{Z}^{45}=g_{Z}^{54}=\left(\frac{g}{2} c_{W}+y g^{\prime} s_{W}-\hat{y} g^{\prime} s_{W}\right) \sin ^{\prime} \cos \theta^{\prime},
\end{array}
$$

with

$$
\cos \theta^{\prime}=\frac{\left(m_{2}-m_{1}-B^{\prime}\right)}{\sqrt{2 c^{2} v^{2}+\left(m_{2}-m_{1}-B^{\prime}\right)^{2}}}, \quad B^{\prime}=\sqrt{\left(m_{2}-m_{1}\right)^{2}+2 c^{2} v^{2}} .
$$


Open Access. This article is distributed under the terms of the Creative Commons Attribution License which permits any use, distribution and reproduction in any medium, provided the original author(s) and source are credited.

\section{References}

[1] ATLAS collaboration, Observation of a new particle in the search for the standard model Higgs boson with the ATLAS detector at the LHC, Phys. Lett. B 716 (2012) 1 [arXiv: 1207.7214] [INSPIRE].

[2] CMS collaboration, Observation of a new boson at a mass of $125 \mathrm{GeV}$ with the CMS experiment at the LHC, Phys. Lett. B 716 (2012) 30 [arXiv:1207.7235] [INSPIRE].

[3] T. Corbett, O. Eboli, J. Gonzalez-Fraile and M. Gonzalez-Garcia, Constraining anomalous Higgs interactions, Phys. Rev. D 86 (2012) 075013 [arXiv:1207.1344] [INSPIRE].

[4] P.P. Giardino, K. Kannike, M. Raidal and A. Strumia, Is the resonance at $125 \mathrm{GeV}$ the Higgs boson?, Phys. Lett. B 718 (2012) 469 [arXiv:1207.1347] [INSPIRE].

[5] J. Espinosa, C. Grojean, M. Muhlleitner and M. Trott, First Glimpses at Higgs' face, JHEP 12 (2012) 045 [arXiv: 1207.1717] [INSPIRE].

[6] ATLAS collaboration, Combined search for the Standard Model Higgs boson in pp collisions at $\sqrt{s}=7 \mathrm{TeV}$ with the ATLAS detector, Phys. Rev. D 86 (2012) 032003 [arXiv:1207.0319] [INSPIRE].

[7] CMS collaboration, Combined results of searches for the standard model Higgs boson in pp collisions at $\sqrt{s}=7 \mathrm{TeV}$, Phys. Lett. B 710 (2012) 26 [arXiv:1202.1488] [INSPIRE].

[8] CMS collaboration, Evidence for a new state decaying into two photons in the search for the standard model Higgs boson in pp collisions, CMS-PAS-HIG-12-015 (2012).

[9] CMS collaboration, Observation of a new boson with a mass near $125 \mathrm{GeV}$, CMS-PAS-HIG-12-020 (2012).

[10] ATLAS collaboration, Observation of an excess of events in the search for the Standard Model Higgs boson in the gamma-gamma channel with the ATLAS detector, ATLAS-CONF-2012-091 (2012).

[11] Tevatron New Physics Higgs Working Group, CDF, D0 collaborations, Updated Combination of CDF and DO Searches for Standard Model Higgs Boson Production with up to $10.0 \mathrm{fb}^{-1}$ of Data, arXiv:1207.0449 [INSPIRE].

[12] CMS collaboration, Search for the standard model Higgs boson decaying into two photons in pp collisions at $\sqrt{s}=7$ TeV, Phys. Lett. B 710 (2012) 403 [arXiv:1202.1487] [InSPIRE].

[13] ATLAS collaboration, Search for the Standard Model Higgs boson in the diphoton decay channel with $4.9 \mathrm{fb}^{-1}$ of $\mathrm{pp}$ collisions at $\sqrt{\mathrm{s}}=7 \mathrm{TeV}$ with ATLAS, Phys. Rev. Lett. 108 (2012) 111803 [arXiv:1202.1414] [INSPIRE].

[14] K. Kumar, R. Vega-Morales and F. Yu, Effects from New Colored States and the Higgs Portal on Gluon Fusion and Higgs Decays, Phys. Rev. D 86 (2012) 113002 [arXiv: 1205.4244] [INSPIRE].

[15] S. Dawson and E. Furlan, A Higgs Conundrum with Vector Fermions, Phys. Rev. D 86 (2012) 015021 [arXiv: 1205.4733] [INSPIRE]. 
[16] N. Bonne and G. Moreau, Reproducing the Higgs boson data with vector-like quarks, Phys. Lett. B 717 (2012) 409 [arXiv: 1206.3360] [INSPIRE].

[17] M. Carena, I. Low and C.E. Wagner, Implications of a Modified Higgs to Diphoton Decay Width, JHEP 08 (2012) 060 [arXiv: 1206.1082] [INSPIRE].

[18] A. Joglekar, P. Schwaller and C.E. Wagner, Dark Matter and Enhanced Higgs to Di-photon Rate from Vector-like Leptons, JHEP 12 (2012) 064 [arXiv:1207.4235] [INSPIRE].

[19] N. Arkani-Hamed, K. Blum, R.T. D'Agnolo and J. Fan, 2:1 for Naturalness at the LHC?, JHEP 01 (2013) 149 [arXiv:1207.4482] [INSPIRE].

[20] L.G. Almeida, E. Bertuzzo, P.A. Machado and R.Z. Funchal, Does $H \rightarrow \gamma \gamma$ Taste like vanilla New Physics?, JHEP 11 (2012) 085 [arXiv: 1207. 5254] [INSPIRE].

[21] H. Davoudiasl, H.-S. Lee and W.J. Marciano, Dark Side of Higgs Diphoton Decays and Muon g-2, Phys. Rev. D 86 (2012) 095009 [arXiv:1208.2973] [InSPIRE].

[22] M. Voloshin, CP Violation in Higgs Diphoton Decay in Models with Vectorlike Heavy Fermions, Phys. Rev. D 86 (2012) 093016 [arXiv: 1208.4303] [INSPIRE].

[23] J. Kearney, A. Pierce and N. Weiner, Vectorlike Fermions and Higgs Couplings, Phys. Rev. D 86 (2012) 113005 [arXiv:1207.7062] [INSPIRE].

[24] H.M. Lee, M. Park and W.-I. Park, Axion-mediated dark matter and Higgs diphoton signal, JHEP 12 (2012) 037 [arXiv: 1209.1955] [INSPIRE].

[25] P. Draper and D. McKeen, Diphotons from Tetraphotons in the Decay of a 125 GeV Higgs at the LHC, Phys. Rev. D 85 (2012) 115023 [arXiv:1204.1061] [INSPIRE].

[26] A. Akeroyd and S. Moretti, Enhancement of $H \rightarrow \gamma \gamma$ from doubly charged scalars in the Higgs Triplet Model, Phys. Rev. D 86 (2012) 035015 [arXiv:1206.0535] [INSPIRE].

[27] L. Wang and X.-F. Han, $130 \mathrm{GeV}$ gamma-ray line and enhancement of $h \rightarrow \gamma \gamma$ in the Higgs triplet model plus a scalar dark matter, Phys. Rev. D 87 (2013) 015015 [arXiv:1209.0376] [INSPIRE].

[28] D. Bertolini and M. McCullough, The Social Higgs, JHEP 12 (2012) 118 [arXiv:1207.4209] [INSPIRE].

[29] E.J. Chun, H.M. Lee and P. Sharma, Vacuum Stability, Perturbativity, EWPD and Higgs-to-diphoton rate in Type II Seesaw Models, JHEP 11 (2012) 106 [arXiv:1209.1303] [INSPIRE].

[30] W.-F. Chang, J.N. Ng and J.M. Wu, Constraints on New Scalars from the LHC $125 \mathrm{GeV}$ Higgs Signal, Phys. Rev. D 86 (2012) 033003 [arXiv:1206.5047] [InSPIRE].

[31] G.D. Kribs and A. Martin, Enhanced di-Higgs Production through Light Colored Scalars, Phys. Rev. D 86 (2012) 095023 [arXiv:1207.4496] [INSPIRE].

[32] I. Dorsner, S. Fajfer, A. Greljo and J.F. Kamenik, Higgs Uncovering Light Scalar Remnants of High Scale Matter Unification, JHEP 11 (2012) 130 [arXiv:1208.1266] [INSPIRE].

[33] A. Urbano, Higgs decay into photons through a spin-2 loop, arXiv:1208.5782 [INSPIRE].

[34] J. Cao, Z. Heng, T. Liu and J.M. Yang, Di-photon Higgs signal at the LHC: a comparative study for different supersymmetric models, Phys. Lett. B 703 (2011) 462 [arXiv:1103.0631] [INSPIRE]. 
[35] J.-J. Cao, Z.-X. Heng, J.M. Yang, Y.-M. Zhang and J.-Y. Zhu, A SM-like Higgs near $125 \mathrm{GeV}$ in low energy SUSY: a comparative study for MSSM and NMSSM, JHEP 03 (2012) 086 [arXiv: 1202.5821] [INSPIRE].

[36] M. Carena, S. Gori, N.R. Shah and C.E. Wagner, A $125 \mathrm{GeV}$ SM-like Higgs in the MSSM and the $\gamma \gamma$ rate, JHEP 03 (2012) 014 [arXiv: 1112.3336] [INSPIRE].

[37] M. Carena, S. Gori, N.R. Shah, C.E. Wagner and L.-T. Wang, Light Stau Phenomenology and the Higgs $\gamma \gamma$ Rate, JHEP 07 (2012) 175 [arXiv:1205.5842] [INSPIRE].

[38] R. Sato, K. Tobioka and N. Yokozaki, Enhanced Diphoton Signal of the Higgs Boson and the Muon g-2 in Gauge Mediation Models, Phys. Lett. B 716 (2012) 441 [arXiv:1208.2630] [INSPIRE].

[39] F. Boudjema and G.D. La Rochelle, Supersymmetric Higgses beyond the MSSM: an update with flavour and Dark Matter constraints, Phys. Rev. D 86 (2012) 115007 [arXiv: 1208.1952] [INSPIRE].

[40] T. Kitahara, Vacuum Stability Constraints on the Enhancement of the $h \rightarrow \gamma \gamma$ rate in the MSSM, JHEP 11 (2012) 021 [arXiv: 1208.4792] [INSPIRE].

[41] K. Schmidt-Hoberg and F. Staub, Enhanced $h \rightarrow \gamma \gamma$ rate in MSSM singlet extensions, JHEP 10 (2012) 195 [arXiv: 1208.1683] [INSPIRE].

[42] N. Haba, K. Kaneta, Y. Mimura and R. Takahashi, Enhancement of Higgs to diphoton decay width in non-perturbative Higgs model, Phys. Lett. B 718 (2013) 1441 [arXiv:1207.5102] [INSPIRE].

[43] B. Bellazzini, C. Petersson and R. Torre, Photophilic Higgs from sgoldstino mixing, Phys. Rev. D 86 (2012) 033016 [arXiv: 1207.0803] [INSPIRE].

[44] A. Alves et al., Probing 3-3-1 Models in Diphoton Higgs Boson Decay, Phys. Rev. D 84 (2011) 115004 [arXiv:1109.0238] [INSPIRE].

[45] D. Carmi, A. Falkowski, E. Kuflik, T. Volansky and J. Zupan, Higgs After the Discovery: A Status Report, JHEP 10 (2012) 196 [arXiv:1207.1718] [INSPIRE].

[46] A. Kobakhidze, Standard Model with a distorted Higgs sector and the enhanced Higgs diphoton decay rate, arXiv: 1208.5180 [INSPIRE].

[47] P. Olesen, $W$ condensation at the LHC?, arXiv:1207.7045 [INSPIRE].

[48] D.S. Alves, P.J. Fox and N.J. Weiner, Higgs Signals in a Type I 2HDM or with a Sister Higgs, arXiv: 1207.5499 [INSPIRE].

[49] M. Reece, Vacuum Instabilities with a Wrong-Sign Higgs-Gluon-Gluon Amplitude, arXiv: 1208.1765 [INSPIRE].

[50] J.R. Ellis, M.K. Gaillard and D.V. Nanopoulos, A Phenomenological Profile of the Higgs Boson, Nucl. Phys. B 106 (1976) 292 [InSPIRE].

[51] O. Eberhardt et al., Impact of a Higgs boson at a mass of $126 \mathrm{GeV}$ on the standard model with three and four fermion generations, Phys. Rev. Lett. 109 (2012) 241802 [arXiv: 1209.1101] [INSPIRE].

[52] O. Eboli, C. Savoy and R. Zukanovich Funchal, A Rationale for Long-lived Quarks and Leptons at the LHC: Low Energy Flavour Theory, JHEP 02 (2012) 123 [arXiv:1112.5108] [INSPIRE]. 
[53] G. Altarelli and R. Barbieri, Vacuum polarization effects of new physics on electroweak processes, Phys. Lett. B 253 (1991) 161 [INSPIRE].

[54] M.E. Peskin and T. Takeuchi, Estimation of oblique electroweak corrections, Phys. Rev. D 46 (1992) 381 [INSPIRE].

[55] M. Baak et al., The Electroweak Fit of the Standard Model after the Discovery of a New Boson at the LHC, Eur. Phys. J. C 72 (2012) 2205 [arXiv:1209.2716] [InSPIRE].

[56] J. Alwall, M. Herquet, F. Maltoni, O. Mattelaer and T. Stelzer, MadGraph 5: Going Beyond, JHEP 06 (2011) 128 [arXiv:1106.0522] [InSPIRE].

[57] J. Pumplin et al., New generation of parton distributions with uncertainties from global QCD analysis, JHEP 07 (2002) 012 [hep-ph/0201195] [INSPIRE].

[58] V.D. Barger et al., Superheavy quarkonium production and decays: a new Higgs signal, Phys. Rev. D 35 (1987) 3366 [Erratum ibid. D 38 (1988) 1632] [INSPIRE].

[59] Y. Kats and M.J. Strassler, Probing Colored Particles with Photons, Leptons and Jets, JHEP 11 (2012) 097 [arXiv: 1204.1119] [InSPIRE].

[60] CDF collaboration, T. Aaltonen et al., Search for new particles decaying into dijets in proton-antiproton collisions at $\sqrt{s}=1.96 \mathrm{TeV}$, Phys. Rev. D 79 (2009) 112002 [arXiv: 0812.4036] [INSPIRE].

[61] CMS collaboration, Search for heavy, top-like quark pair production in the dilepton final state in pp collisions at $\sqrt{s}=7 \mathrm{TeV}$, Phys. Lett. B 716 (2012) 103 [arXiv:1203.5410] [INSPIRE].

[62] K. Rao and D. Whiteson, Triangulating an exotic T quark, Phys. Rev. D 86 (2012) 015008 [arXiv: 1204.4504] [INSPIRE].

[63] CMS collaboration, Search for heavy bottom-like quarks in 4.9 inverse femtobarns of pp collisions at $\sqrt{s}=7 \mathrm{TeV}$, JHEP 05 (2012) 123 [arXiv:1204.1088] [INSPIRE].

[64] CMS collaboration, Search for stopped long-lived particles produced in pp collisions at $\sqrt{s}=7 \mathrm{TeV}$, JHEP 08 (2012) 026 [arXiv: 1207.0106] [INSPIRE].

[65] CMS collaboration, Search for heavy long-lived charged particles in pp collisions at $\sqrt{s}=7 \mathrm{TeV}$, Phys. Lett. B 713 (2012) 408 [arXiv: 1205.0272] [inSPIRE].

[66] CMS collaboration, Measurement of WW production rate, CMS-PAS-SMP-12-013 (2013).

[67] ATLAS collaboration, Coupling properties of the new Higgs-like boson observed with the ATLAS detector at the LHC, ATLAS-CONF-2012-127 (2012). 